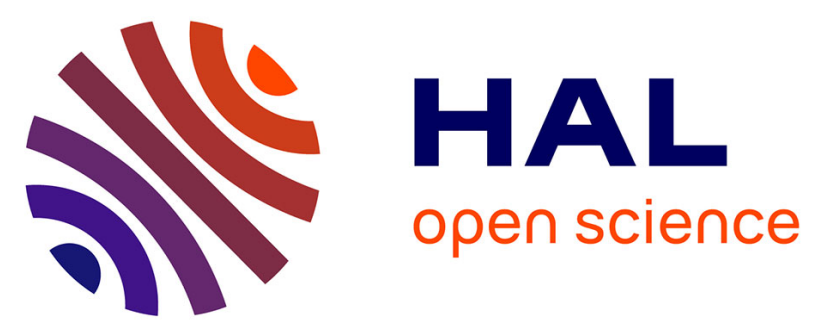

\title{
Comment réduire les incertitudes d'estimation des quantiles de débits des crues exceptionnelles? Comparaison d'approches statistiques historiques et/ou régionales
}

Chi Cong Nguyen, Olivier Payrastre, Eric Gaume

\section{To cite this version:}

Chi Cong Nguyen, Olivier Payrastre, Eric Gaume. Comment réduire les incertitudes d'estimation des quantiles de débits des crues exceptionnelles? Comparaison d'approches statistiques historiques et/ou régionales. La Houille Blanche - Revue internationale de l'eau, 2015, 2015 (3), pp. 64-71. 10.1051/lhb/20150035 . hal-01263206

\section{HAL Id: hal-01263206 \\ https://hal.science/hal-01263206}

Submitted on 1 Feb 2016

HAL is a multi-disciplinary open access archive for the deposit and dissemination of scientific research documents, whether they are published or not. The documents may come from teaching and research institutions in France or abroad, or from public or private research centers.
L'archive ouverte pluridisciplinaire HAL, est destinée au dépôt et à la diffusion de documents scientifiques de niveau recherche, publiés ou non, émanant des établissements d'enseignement et de recherche français ou étrangers, des laboratoires publics ou privés. 


\title{
Comment réduire les incertitudes d'estimation des quantiles de débits des crues exceptionnelles? Comparaison d'approches statistiques historiques et/ou régionales
}

\author{
Chi Cong N'GUYEN ${ }^{1,2}$, Olivier PAYRASTRE ${ }^{1}$, Eric GAUME ${ }^{1}$
}

1. Ifsttar, centre de Nantes - Route de bouaye, CS4, 44344 Bouguenais Cedex-prenom.nom@ifsttar.fr

2. Université de Da Nang, 54 Street Nguyen Luong Bang, 555704, Danang city, Vietnam

\begin{abstract}
RÉSUMÉ. - Cet article présente un travail de comparaison entre des approches locales et régionales d'analyse fréquentielle des débits de crues, en mettant l'accent sur l'intérêt de la valorisation de données relatives à des crues extrêmes, et sur les effets associés aux hypothèses des analyses régionales (homogénéité statistique des séries et indépendance des observations). Quatre approches sont comparées à travers deux études de cas (région de l'Ardèche et du Var) : 1 et 2 - analyses locales avec ou sans données historiques, 3 - analyse régionale classique (index de crue), basée sur les séries hydrométriques, 4 - analyse régionale avec mobilisation des crues extrêmes (historiques ou récentes sur sites non jaugés). Le cadre d'inférence utilisé (distribution GEV et approche Bayésienne) permet de représenter facilement l'effet des jeux de données sur la précision des quantiles estimés. Le travail de comparaison mené repose à la fois sur les données réelles et sur des simulations Monte Carlo, qui permettent de s'affranchir des effets de l'échantillonnage et d'analyser les effets possibles d'hétérogénéités régionales. Les résultats présentés indiquent que même un faible niveau d'hétérogénéité régionale, qui ne peut être détecté par les tests d'homogénéité généralement mis en œuvre, est susceptible de limiter fortement l'intérêt des approches régionales classiques. En revanche, les informations, même imprécises, sur les crues majeures historiques ou ayant affecté des bassins versant non jaugés, permettent en général de réduire significativement les incertitudes d'estimation statistique. On peut regretter que ce type d'information soit encore aujourd'hui trop peu exploité.
\end{abstract}

Mots-clés : Crue, Statistique, Analyses régionales, Méthodes Bayesiennes

\section{Reducing uncertainties on low-probability flood peak discharge quantile estimates: comparison of historical and/or regional approaches}

\begin{abstract}
This paper presents a detailed comparison of several local and regional flood frequency analysis approaches, with a special focus on the effects of 1 - the availability of information on extreme floods (historical data or floods observed at ungauged sites), and 2 - the assumptions associated with regional approaches (statistical homogeneity of considered series, independency between observations). Four flood frequency estimation approaches are compared: 1 - local analysis, 2 - local analysis with historical information, 3 - regional index-flood analysis based on stream gauge series, 4 - regional analysis involving information on extremes (including both historical floods and recent floods observed at ungauged sites). The inference approach used - GEV distribution and Bayesian approach for parameter estimation - enables to represent the effects of the analysed datasets on the accuracy of the estimated quantiles. The results presented are based on two case studies: the Ardèche and Var regions in south east of France. The comparison relies both (1) available observed datasets, and (2) Monte Carlo simulations in order to generalize the conclusions and to simulate the effects of possible regional heterogeneities. The obtained results indicate that even a relatively limited level of heterogeneity, that may not be detected by classical homogeneity tests, may highly affect the performances of the regional approaches. However, the information on extreme floods, historical floods or recent estimated extremes at ungauged sites, generally enable to drastically reduce the uncertainties of statistical estimations. As far as possible, gathering of such information and incorporating it into flood frequency studies should be promoted.
\end{abstract}

Key-words: Flood, Frequency, Regional analyses, Bayesian method

\section{INTRODUCTION}

L'analyse fréquentielle des débits des crues repose classiquement sur les séries d'observations des stations hydrométriques. Ces séries s'avèrent souvent trop courtes - quelques dizaines d'années en général - pour permettre une estimation précise des valeurs des quantiles rares, notamment ceux de période de retour supérieure à cent ans. La réduction des incertitudes d'estimation passe soit par la fixation de contraintes sur les formes des distributions statistiques, fondées sur des hypothèses (méthodes de simulation pluie-débit, méthode du Gradex), soit par une augmentation des effectifs des échantillons étudiés. Deux grandes familles d'approches existent pour enrichir les séries de données : 1) les approches historiques, valorisant les informations disponibles sur les crues anciennes du site étudié, antérieures à la période de mesure systématique des débits, et 2) les approches régionales consistant à fusionner des données issues de plusieurs 
sites considérés comme homogènes d'un point de vue statistique, pour constituer de larges échantillons régionaux. Cet article enrichit et complète les travaux récents publiés par les auteurs [N'guyen et al., 2013 ; Payrastre et al., 2011, 2013], en illustrant à la fois l'intérêt et les limites de ces approches régionales, par comparaison à des approches locales mobilisant ou non des informations historiques. Les exemples fournis concernent essentiellement l'estimation des quantiles de débits centennaux qui peuvent être estimés avec un niveau d'incertitude raisonnable compte-tenu des données disponibles. Mais les tendances concernant les estimations de quantiles de périodes de retour plus élevées sont identiques (cf. Tableau 3 présentant des estimations de quantiles millénaux) dans la mesure où ils sont calculés à partir des mêmes paramètres de loi de probabilité calés sur les données disponibles. Les illustrations de cet article s'appuient sur deux études de cas : les bassins de l'Ardèche et de l'Argens dans le Var. Une question particulière a guidé cette étude : dans la mesure où il est impossible de garantir la parfaite homogénéité statistique des séries régionales, comment s'équilibrent l'effet bénéfique de l'augmentation des effectifs et l'effet perturbateur lié au mélange de données hétérogènes dans le cas des analyses régionales ? Ne perd-on pas d'un côté ce que l'on a gagné de l'autre?

Quatre méthodes d'estimation sont comparées : 1) analyse de la série hydrométrique locale, 2) analyse locale complétée par les informations historiques, 3) analyse régionale classique sur le modèle proposé par Hosking \& Wallis [1997], fondée sur les séries hydrométriques, 4) analyse régionale modifiée intégrant les valeurs de débits extrêmes estimées, dans la région, sur des sites non jaugés [Gaume et al., 2010 ;N'guyen et al., 2013]. La méthode d'inférence statistique utilisée repose sur une distribution GEV et une approche Bayésienne MCMC pour l'estimation des paramètres, permettant de calculer des intervalles de crédibilité autour des valeurs de quantiles estimées.

Afin de compléter l'étude des séries de données réelles disponibles pour chaque étude de cas, et en particulier pour s'affranchir des effets de l'échantillonnage et évaluer le caractère générique des conclusions qui peuvent être tirées, la même analyse est reconduite sur un large jeu d'échantillons de données synthétiques, générés par tirage aléatoire de type Monte Carlo et ayant les mêmes caractéristiques que les échantillons disponibles (type de distribution statistique, nombre de sites, effectifs des échantillons, hétérogénéité régionale).

L'article est organisé de la façon suivante : le paragraphe II détaille les 4 approches d'inférence comparées, ainsi que le principe des simulations Monte Carlo effectuées, destinées à s'affranchir des effets de l'échantillonnage et à simuler différents niveaux d'hétérogénéité des échantillons régionaux ; le paragraphe III présente les deux études de cas utilisées, les jeux de données disponibles et les choix effectués dans chaque cas pour les simulations; le paragraphe IV présente et discute les résultats des simulations.

\section{METHODOLOGIE}

\section{II.1. Principe général des approches d'inférence comparées}

L'approche d'inférence mise en œuvre repose sur l'utilisation d'une distribution candidate de type GEV. Outre la simplicité des calculs, cette distribution à 3 paramètres présente l'avantage d'une certaine sensibilité qui permet de mettre facilement en évidence les effets liées aux jeux de données valorisées. Les tendances identifiées ici devraient en toute logique se retrouver, de façon plus ou moins marquée, avec d'autres formes de distributions. Les paramètres $\theta=(\xi, \alpha, \kappa)$ de la distribution sont évalués ici par une approche Bayésienne désormais relativement répandue en hydrologie [Reis et Stedinger, 2005 ; Renard et al., 2006 ; Neppel et al., 2010 ; Gaume et al., 2010 ; Payrastre et al., 2011, 2013 ; N'guyen et al., 2013], et qui consiste à déterminer la distribution de probabilité $\mathrm{p}(\theta \mid \mathrm{D})$ des paramètres conditionnellement au jeu d'observations disponibles $\mathrm{D}$, par une application directe de la formule de Bayes L'échantillonnage de paramètres dans la distribution $\mathrm{p}(\theta \mid \mathrm{D})$ est effectué via un algorithme MCMC. Cette approche permet d'obtenir des intervalles de crédibilité associés aux paramètres et aux quantiles de crue estimés. Dans la suite de l'article, les intervalles présentés seront des intervalles à $90 \%$ obtenus sur le quantile centennal, complétés par quelques résultats sur les quantiles millénaux.

Aucune information a priori n'étant considérée ici (prior uniforme), la probabilité $\mathrm{p}(\theta \mid \mathrm{D})$ est directement proportionnelle à la vraisemblance des observations $\mathrm{L}(\mathrm{D} \mid \theta)$, dont une expression générique peut être donnée par la formule suivante :

$$
\begin{gathered}
L(D / \theta)=\prod_{i=1}^{s}\left[\prod_{j=1}^{n_{i, 0}}\left[F_{\theta}\left(\frac{Q_{i, j}^{U}}{\mu_{i}}\right)-F_{\theta}\left(\frac{Q_{i, j}^{L}}{\mu_{i}}\right)\right]\right] \\
\prod_{i=1}^{s}\left[\prod_{k=1}^{h_{i}}\left[F_{\theta}\left(\frac{Q_{i, k}^{U}}{\mu_{i}}\right)-F_{\theta}\left(\frac{Q_{i, k}^{L}}{\mu_{i}}\right)\right] \cdot \prod_{k=1}^{h_{i}}\left[F_{\theta}\left(\frac{Q_{i, k}^{S}}{\mu_{i}}\right)\right]^{\left(n_{i, k}-1\right)}\right]
\end{gathered}
$$

en considérant un jeu de données $\mathrm{D}$ recueillies sur $\mathrm{s}$ sites différents, i représentant l'indice du site et $\mu_{\mathrm{i}}$ la valeur de l'index de crue associée [Dalrymple, 1960]. Les données disponibles sur chaque site peuvent être constituées :

- d'une série d'enregistrements continus issus d'une station hydrométrique, incluant $n_{i, 0}$ valeurs maximales annuelles de débit, chacune étant comprise dans un intervalle $\left[\mathrm{Q}_{\mathrm{i}, \mathrm{j}}{ }^{\mathrm{L}}, \mathrm{Q}_{\mathrm{i}, \mathrm{j}}{ }^{\mathrm{U}}\right]$ $\left(\mathrm{j}=\left[1, \ldots, \mathrm{n}_{\mathrm{i}, 0}\right]\right)$. Un intervalle de $+-1 \%$ autour de la valeur mesurée $\mathrm{Q}_{\mathrm{i}, \mathrm{j}}$ a systématiquement été considéré ici.

- et/ou $h_{i}$ crues dont le débit maximum est compris dans un intervalle $\left[\mathrm{Q}_{\mathrm{i}, \mathrm{k}}{ }^{\mathrm{L}}, \mathrm{Q}_{\mathrm{i}, \mathrm{k}}{ }^{\mathrm{U}}\right]\left(\mathrm{k}=\left[1, \ldots, \mathrm{h}_{\mathrm{i}}\right]\right)$, chacune de ces crues étant la seule à avoir dépassé un seuil de perception de débit $\mathrm{Q}_{\mathrm{i}, \mathrm{k}}{ }^{\mathrm{s}}$ sur une période de $\mathrm{n}_{\mathrm{i}, \mathrm{k}}$ années. Ces crues peuvent indifféremment être des crues historiques ou des crues extrêmes récentes enregistrées sur des sites non jaugés. Dans ce cas, les intervalles $\left[\mathrm{Q}_{\mathrm{i}, \mathrm{k}}{ }^{\mathrm{L}}, \mathrm{Q}_{\mathrm{i}, \mathrm{k}}{ }^{\mathrm{U}}\right]$ sont déterminés au cas par cas en fonction des informations disponibles. En l'absence d'informations, des intervalles de $+-1 \%$ ont également été considérés ici.

Les quatre approches qui seront comparées par la suite diffèrent finalement par le contenu du jeu du jeu de données D valorisé dans chaque cas. Les situations suivantes ont été distinguées :

- Cas $\mathrm{n}^{\circ} 1$ : Analyse locale. Cette approche se base uniquement sur la série hydrométrique du site considéré. On a alors $\mathrm{s}=1$ (site unique) et $\mu_{1}=1$ (pas de normalisation de la série par l'index de crue), et $\mathrm{h}_{1}=0$ (pas de crues historiques).

- Cas $\mathrm{n}^{\circ} 2$ : Analyse locale avec données historiques. Par rapport à la précédente, cette approche ajoute une série de crues historiques connues localement : $h_{1} \neq 0$. 
- Cas $\mathrm{n}^{\circ} 3$ : Analyse régionale classique. Cette approche correspond à celle développée par Hosking et Wallis [1986]. Dans ce cas, l'information se limite a des séries d'enregistrements hydrométriques disponibles sur s sites, sans information sur les extrêmes $\left(h_{i}=0\right.$ quel que soit $\left.i\right)$. Les index de crue $\mu_{\mathrm{i}}$ correspondent ici à la moyenne de chaque série $\mathrm{Q}_{\mathrm{ij}}\left(\mathrm{j}=\left[1, \ldots, \mathrm{n}_{\mathrm{i}, 0}\right]\right)$.

- Cas $\mathrm{n}^{\circ} 4$ : Analyse régionale avec crues extrêmes. Cette approche a initialement été proposée par Gaume et al. [2010]. Elle combine des séries hydrométriques sur sites jaugés, avec des données historiques associées et/ou des crues extrêmes recensées sur des sites non jaugés. De façon à pouvoir valoriser ces informations relatives à des sites non jaugés, dans cette approche le calcul de l'index de crue $\mu_{i}$ repose sur une relation d'index de crue du type $\mu_{\mathrm{i}}=\mathrm{S}_{\mathrm{i}}^{\beta}, \mathrm{S}_{\mathrm{i}}$ étant la surface du bassin versant amont du site $i$, et $\beta$ un paramètre à estimer.

Des descriptions plus détaillées de ces différentes approches pourront être trouvés dans Neppel et al. [2010] et Payrastre et al. [2006, 2011, 2013] concernant les approches locales avec ou sans données historiques, et dans Gaume et al. [2010] et N'guyen et al. [2013] pour les approches régionales.

\section{II.2. Principe des simulations Monte Carlo effectuées}

Outre l'application directe des quatre approches décrites au paragraphe précédent, à partir des jeux de données disponibles dans les différentes études de cas, le travail de comparaison présenté par la suite repose sur des jeux de données simulées, dont les caractéristiques (nombre de sites, nombre et durées des séries hydrométriques, nombre de crues extrêmes et périodes associées aux différents seuils de perception, etc ..), reprennent celles des jeux de données réels. Ces simulations ont pour objectif de s'affranchir des effets de l'échantillonnage, et de dégager ainsi des conclusions de portée plus générale que celles obtenues à partir d'un échantillon unique. Elles permettent également d'aborder les questions suivantes : (i) confirmer la validité des intervalles de crédibilité à $90 \%$ obtenus avec l'approche Bayésienne, et (ii) examiner la sensibilité des résultats aux hypothèses des approches régionales, notamment à la présence d'hétérogénéités des séries au sein des régions considérées. Les approches régionales reposent également sur une hypothèse d'indépendance des observations souvent difficile à garantir : l'impact possible de la présence d'une corrélation spatiale des observations n'a pas été testé dans les simulations, mais sera abordé au travers des études de cas (cas de l'Argens).

Le principe des simulations est le suivant : 1000 échantillons de données régionales sont simulés à partir d'une distribution GEV régionale et d'une relation d'index de crue $\left(\mu_{i}=S_{i}^{\beta}\right)$ toutes deux ajustées à partir des données réelles. Les quatre approches sont ensuite appliquées à chaque jeu de données simulé. Le mode de la distribution a posteriori (ou maximum de vraisemblance) sur le quantile centennal $\widehat{Q}_{100}^{M L}$ et l'intervalle de crédibilité à $90 \%$ correspondant $\left[\widehat{Q}_{100}^{5}, \widehat{Q}_{100}^{95}\right]$ sont calculés pour chaque site au sein de la région. Le résultat final est un jeu de 1000 valeurs de débits centennaux estimés avec dans chaque cas l'intervalle de crédibilité à $90 \%$ associé.

\section{II.2.1. Dispersion des quantiles centennaux estimés}

Dans un premier temps seules les valeurs $\widehat{Q}_{100}^{M L}$ sont examinées, et leur dispersion autour de la vraie valeur du quantile $Q_{100}$ à estimer est représentée graphiquement (cf. Figure 4). Cette dispersion permet d'obtenir une idée de la précision respective de chacune des approches testées.

\section{II.2.2. Vérification de la validité des intervalles de crédibilité à $90 \%$}

Cette vérification est effectuée en calculant la position du véritable quantile centennal $Q_{100}$ au sein de chacune des distributions a posteriori estimées : $\widehat{F}_{Q_{100}}\left(Q_{100}\right)$. Si les distributions a posteriori $\widehat{F}_{Q_{100}}$ sont correctement estimées, les valeurs de $\widehat{F}_{Q_{100}}\left(Q_{100}\right)$ obtenues doivent être distribuées uniformément sur les 1000 simulations effectuées (cf. Figure 1). Cette vérification permet finalement d'obtenir une information sur la valeur des intervalles de crédibilité qui seront calculés à partir des échantillons de données réelles.

\section{II.2.3. Introduction d'hétérogénéités autour de la relation d'index de crue}

Les simulations permettent également de tenir compte d'un certain niveau d'hétérogénéité de la région considérée autour de la relation d'index de crue : pour chaque site et chaque échantillon simulé, la valeur théorique $\mu_{i}=S_{i}^{\beta}$ de l'index de crue sur le site i est alors remplacée par une valeur aléatoire tirée dans une distribution log-normale de moyenne $S_{i}^{\beta}$ et d'écart type $\delta \cdot S_{i}^{\beta}$, d pouvant être choisi en fonction des fluctuations constatées des moyennes des séries réelles autour de la relation d'index de crue théorique (cf. Figure 3).

\section{II.2.4. Introduction d'hétérogénéités dans la forme de distributions locales}

Des hétérogénéités peuvent également être introduites dans la forme des distributions GEV considérées sur chaque a)

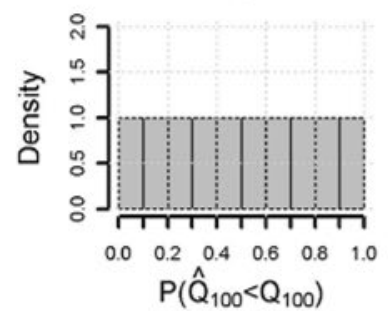

b)

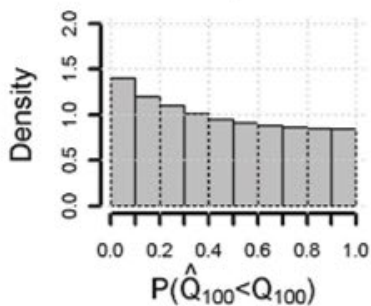

c)

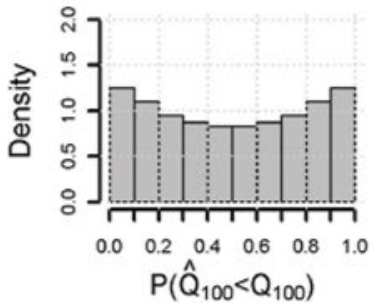

d)

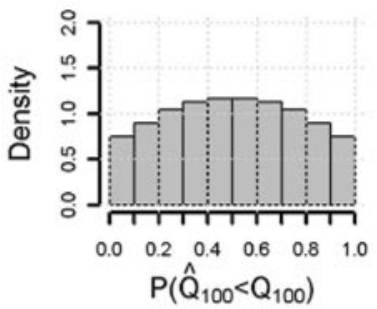

Figure 1 : Résultats possibles du test de validité des intervalles de crédibilité à partir du calcul de $\widehat{F}_{Q_{100}}\left(Q_{100}\right)$ : a) estimation correcte, b) présence de biais (surestimation), c) largeur des intervalles sous-estimée, d) largeur des intervalles surestimée. 
site. Cette hétérogénéité a été simulée ici en générant aléatoirement des fluctuations des L-moments $L_{C V}$ et $L_{s}$ autour des valeurs réelles associées à la distribution GEV utilisée pour les simulations, chaque couple $L_{C V}-L$ ainsi obtenu étant considéré comme représentatif de la forme d'une des distributions locales. Cette procédure comporte deux étapes principales :

(i) pour chaque simulation et chaque site de la région considérée, un couple $L_{C V}-L_{\text {skewness }}$ est généré aléatoirement à partir d'une distribution normale bivariée. Cette distribution normale a été préalablement ajustée en examinant les fluctuations des couples $L_{C V}-L_{\text {skewness }}$ pour des échantillons tirés dans la distribution GEV initiale. Ces fluctuations sont présentées sur la Figure 2 dans le cas d'échantillons de durée respective 40 ans et 20 ans.

(ii) Le couple $L_{C V}-L_{\text {skewness }}$ obtenu permet de calculer les paramètres de la loi GEV appliquée pour générer l'échantillon du site considéré.

(iii) à chaque simulation, le test d'homogénéité proposé par Hosking et Wallis [1993] est appliqué à l'échantillon régional généré, ce qui permet de filtrer une partie de l'hétérogénéité générée. Le nombre d'échantillons finalement retenus pour l'examen des résultats est donc inférieur à 1000 (nombre d'échantillons générés) : il s'élève à 799 pour l'étude de cas de l'Ardèche et 666 pour l'étude de cas de l'Argens (Var). a)

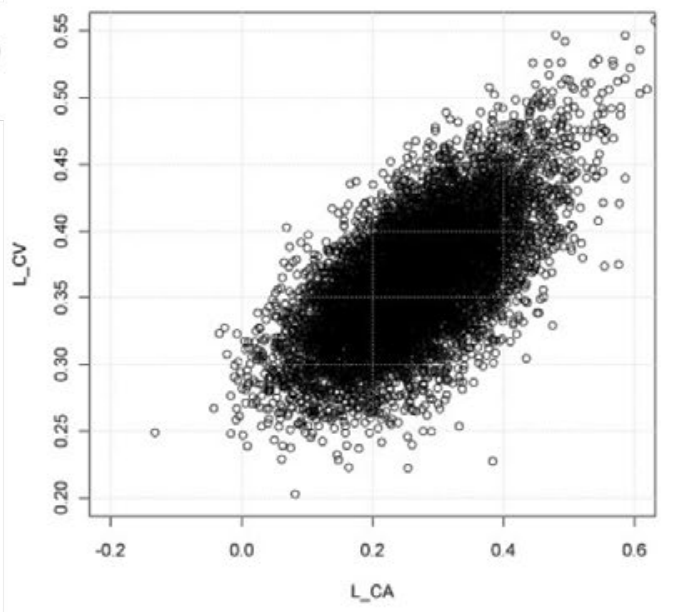

b)

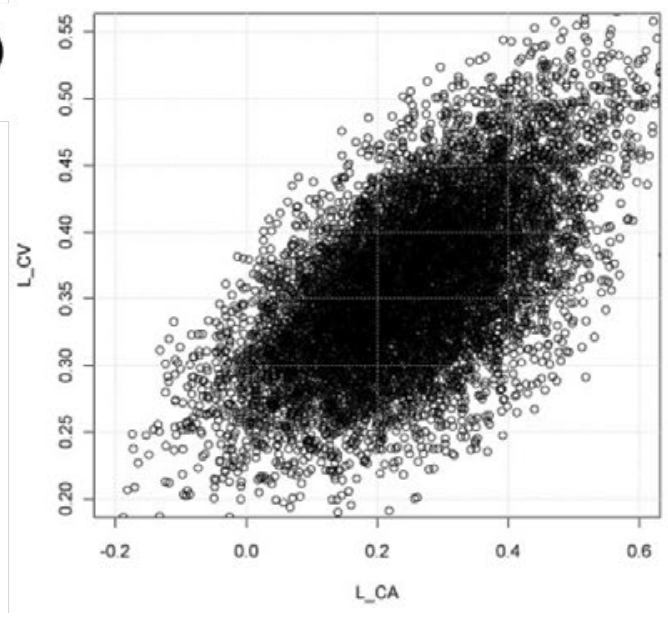

Figure 2 : Exemple des fluctuations des couples $L_{C V}-L_{\text {skewness }}$ pour des échantillons générés à partir d'une même distribution GEV : $a$-échantillons d'une durée de 40 ans, b-échantillons d'une durée de 20 ans. Des fluctuations similaires à celles observées dans le cas a (hétérogénéité modérée) ont été introduites lors des simulations conduites sur les études de cas de l'Ardèche et de l'Argens (Var).

a)

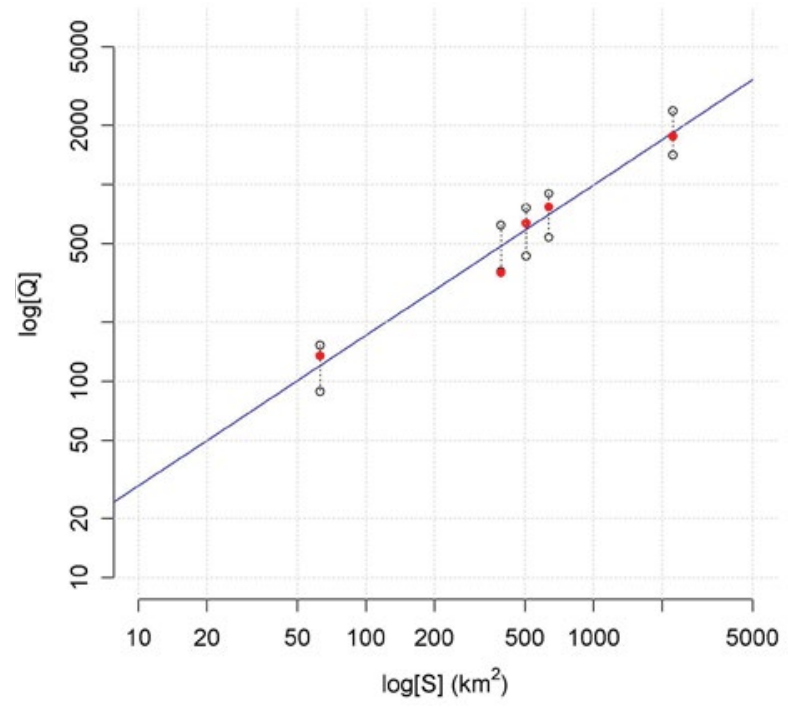

b)

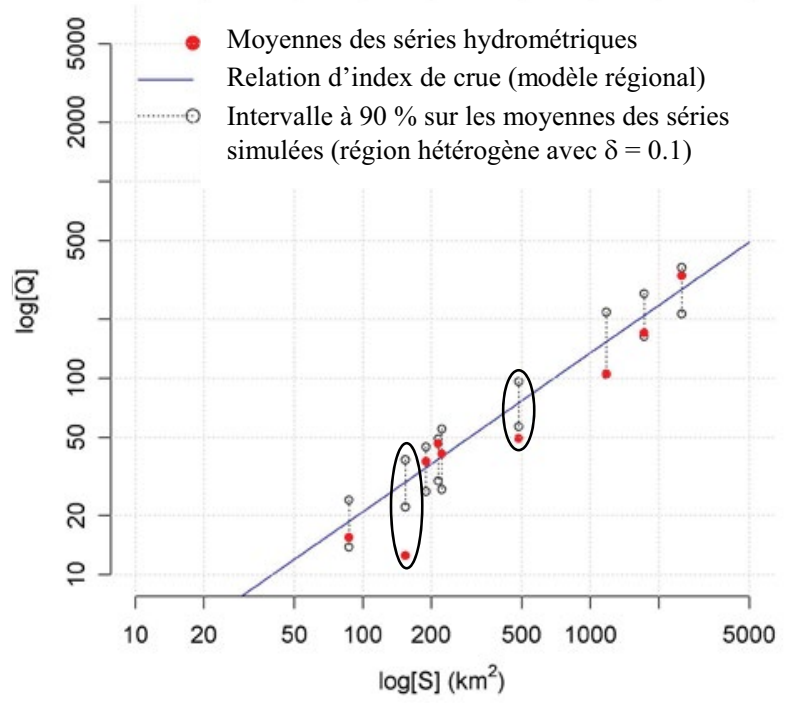

Figure 3 : Comparaison entre les moyennes des échantillons observés aux stations hydrométriques, et les fluctuations des moyennes d'échantillons de même effectif simulés à partir du modèle régional basé sur la relation d'index de crue $\mu_{i}=S_{i}^{\beta}$, avec introduction d'une hétérogénéité autour de cette relation $(\delta=0.1)$ : a) cas de l'Ardèche, b) cas de l'Argens. Dans ce deuxième cas, deux sites (entourés) ont été écartés de la région. 


\section{ETUDES DE CAS PRÉSENTÉES}

\section{III.1. Région de l'Ardèche}

Cette étude de cas a déjà été utilisée dans Gaume et al. [2010] et N'guyen et al. [2013] pour la mise au point de l'approche d'analyse régionale intégrant les crues extrêmes sur sites non jaugés (cas $\left.n^{\circ} 4\right)$. Cette région offre en effet l'avantage de disposer d'un inventaire des crues extrêmes observées sur les 50 dernières années, réalisé dans le cadre du programme Européen Hydrate [Gaume et al., 2009]. La région, qui couvre les bassins versants de l'Ardèche et de l'Eyrieux, comporte par ailleurs 5 stations hydrométriques présentant à la fois une bonne qualité et une continuité des enregistrements, couvrant au total 168 années-stations d'enregistrements.

Deux sites au sein de cette région seront plus particulièrement étudiés ici : celui de la station hydrométrique de Saint Laurent sur la Borne $\left(63 \mathrm{~km}^{2}\right)$, et celui de la station de Sauze-Saint Martin sur le cours principal de l'Ardèche $\left(2240 \mathrm{~km}^{2}\right)$. Ce deuxième site présente l'avantage de l'existence d'une série historique locale. Les caractéristiques des jeux de données valorisables pour chacun des deux sites sont synthétisées dans le Tableau 1.

\section{III.2. Région de l'Argens dans le Var}

Cette étude de cas regroupe le bassin versant de l'Argens et ses différents affluents (le Caramy, l'Issole, le Cauron, le Reyran, l'Aille, et la Nartuby), ainsi qu'un petit bassin contigu : la Siagnole. La région présente la particularité d'avoir été touchée en 2010 par une crue de forte intensité, qui dépasse nettement l'ensemble des crues enregistrées dans les séries hydrométriques, et dont la période de retour s'avère de ce fait délicate à évaluer.

Les séries hydrométriques disponibles dans la région se répartissent sur 9 sites pour un total de 249 années-station. Un site sera plus particulièrement étudié ici : celui de Trans-en-Provence sur la Nartuby $\left(190 \mathrm{~km}^{2}\right)$. Sur ce site, la série hydrométrique locale s'interrompt en 2009 car la station a été emportée par la crue de 2010. La crue de 2010, dont le débit a été estimé a postériori, présente une intensité similaire voire supérieure à deux crues historiques identifiées en 1827 et 1676 . Ces trois crues sont regroupées et assimilées à une série historique d'une durée de 360 années, précédant la série hydrométrique.

Contrairement à l'Ardèche, cette région ne présente pas d'inventaire exhaustif des crues extrêmes recensées dans un passé récent. Néanmoins, le débit de la crue de 2010 s'est avéré particulièrement important sur la Florieye à Taradeau $\left(87 \mathrm{~km}^{2}\right)$, et a pu être estimé a posteriori. Cette crue a été utilisée pour constituer le jeu de données des crues extrêmes sur sites non jaugés, qui comporte par conséquent une crue unique considérée comme la plus forte sur une période de 300 ans. Les caractéristiques du jeu de données finalement valorisé sont synthétisées dans le Tableau 1.

\section{III.3. Estimation du niveau d'hétérogénéité des deux régions considérées}

\section{III.3.1. Forme des distributions locales}

L'hétérogénéité de la forme des distributions locales a été évaluée dans chaque cas en superposant les distributions empiriques calculées pour chaque série locale, et en appliquant le test d'homogénéité proposé par Hosking et Wallis [1993]. Pour les deux régions, les distributions empiriques locales ne paraissent pas véritablement similaires, même si elles restent proches. Par ailleurs les résultats du test d'Hosking et Wallis suggèrent la présence d'une certaine hétérogénéité, en particulier dans le cas de l'Ardèche $(\mathrm{H} 1=2.7)$, mais également dans le cas du Var $(\mathrm{H} 1=1.5)$. Ceci a conduit, dans les simulations qui seront présentées ci-après, à considérer un niveau d'hétérogénéité modéré pour ces deux régions, correspondant au cas a) de la Figure 2.

\section{III.3.2. Relation d'index de crue}

Le niveau d'hétérogénéité autour de la relation d'index de crue peut être estimé graphiquement en superposant les moyennes des échantillons observés sur chaque site, à celles d'échantillons générés à partir du modèle théorique ajusté, dans lequel une part d'hétérogénéité est introduite selon la procédure décrite au paragraphe II.2.3.

La Figure 3 présente les résultats de cette comparaison des moyennes d'échantillons observés et simulés. Dans le cas de l'Ardèche, les fluctuations des moyennes des séries locales sont cohérentes avec un niveau d'hétérogénéité correspondant à $\delta=0.1$. Il en est de même dans le cas de l'Argens, à l'exception de deux sites qui semblent s'écarter plus significativement de la relation moyenne. Ces deux sites ont par conséquent été écartés pour l'application de l'approche régionale basée sur la relation d'index de crue.

\section{RÉSULTATS ET DISCUSSION}

\section{IV.1. Simulations}

Les résultats des simulations sont présentés sur les Figure 4 et Figure 5. Ces résultats illustrent assez logiquement les performances limitées des approches locales basées sur les seules séries hydrométriques (cas $\left.n^{\circ} 1\right)$. La mobilisation de données historiques ou de données sur les crues extrêmes récentes (sites non jaugés), que ce soit dans le cas

Tableau 1 : Caractéristiques des jeux de données valorisées pour les deux régions et trois sites étudiés.

\begin{tabular}{|l|c|c|c|c|c|c|c|c|}
\hline \multicolumn{1}{|c|}{ Site } & $\begin{array}{c}\text { Surface } \\
\text { en } \mathbf{k m}^{2}\end{array}$ & $\begin{array}{c}\text { Série } \\
\text { hydrométrique } \\
\text { locale }\end{array}$ & \multicolumn{2}{|c|}{ Série historique locale } & $\begin{array}{c}\text { Séries hydrométriques } \\
\text { régionales }\end{array}$ & \multicolumn{3}{c|}{$\begin{array}{c}\text { Crues extrêmes } \\
\text { régionales }\end{array}$} \\
\hline & & Durée (années) & $\begin{array}{c}\text { Nombre } \\
\text { de crues }\end{array}$ & $\begin{array}{c}\text { Durée } \\
\text { (années) }\end{array}$ & $\begin{array}{c}\text { Nombre } \\
\text { de sites }\end{array}$ & $\begin{array}{c}\text { Durée } \\
\text { (années) }\end{array}$ & $\begin{array}{c}\text { Nombre } \\
\text { de sites }\end{array}$ & $\begin{array}{c}\text { Durée } \\
\text { (années) }\end{array}$ \\
\hline Saint Laurent & 63 & 30 & 0 & 0 & 4 & 138 & 18 & 900 \\
\hline Saint Martin & 2240 & 43 & 1 & 50 & 4 & 125 & 17 & 850 \\
\hline Trans-en-Provence & 190 & 34 & 3 & 360 & 8 & 215 & 1 & 300 \\
\hline
\end{tabular}


a)

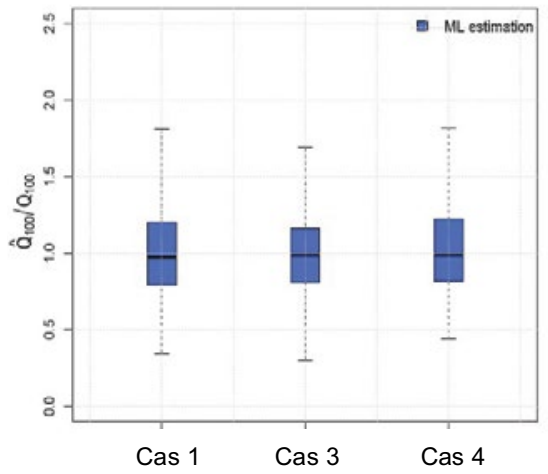

b)

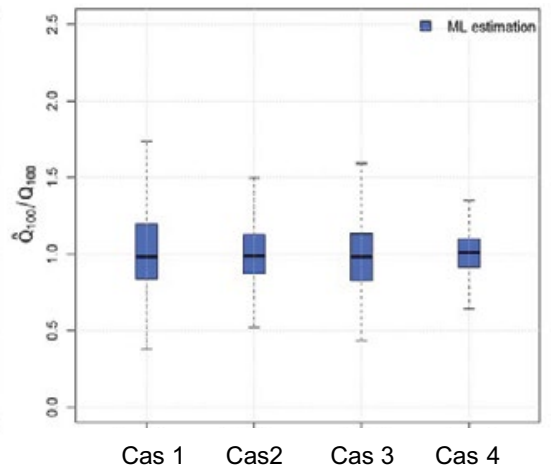

c)

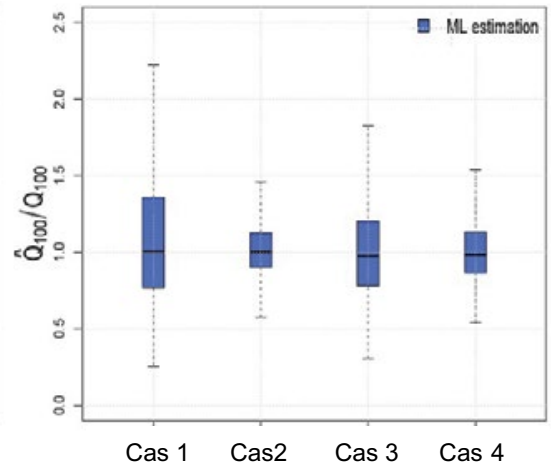

Figure 4 : Distributions des rapports entre quantiles estimés $\widehat{Q}_{i, M L}^{(100)}$ et quantile vrai $Q_{i}^{(100)}$, obtenues par simulations pour les 3 sites présentés dans le tableau 1 : a) Saint Laurent dans l'Ardèche, b) Saint Martin dans l'Ardèche, et c) Trans en Provence dans le Var (Argens). Les 4 cas présentés correspondent aux 4 approches détaillées au paragraphe II.1.

a)

Cas 1

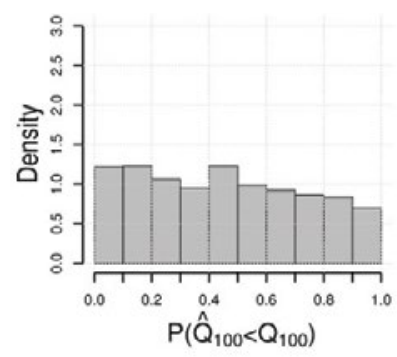

b)

Cas 1

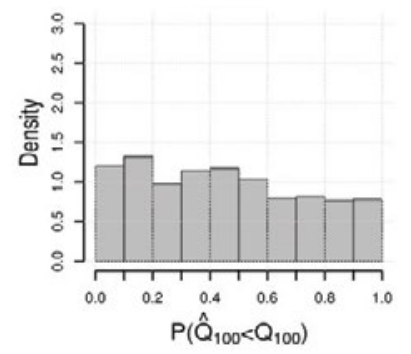

Cas 2

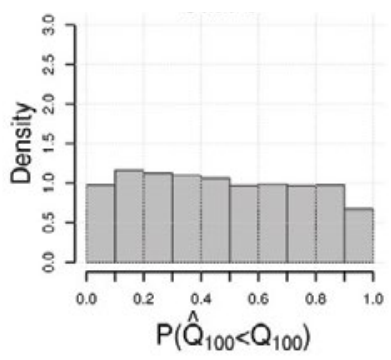

Cas 2

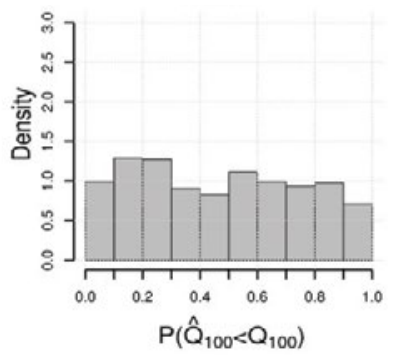

Cas 3

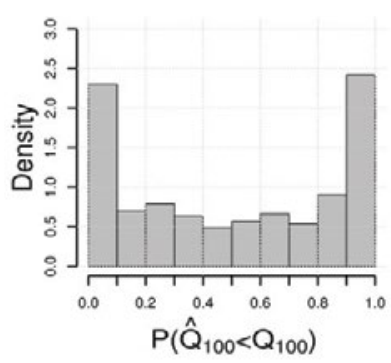

Cas 3

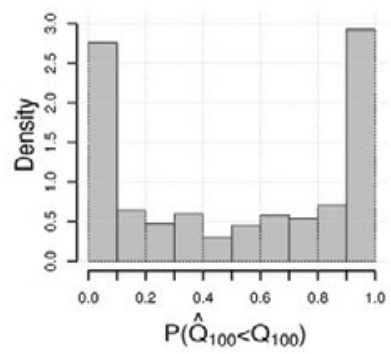

Cas 4

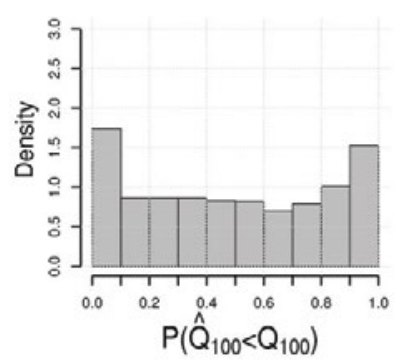

Cas 4

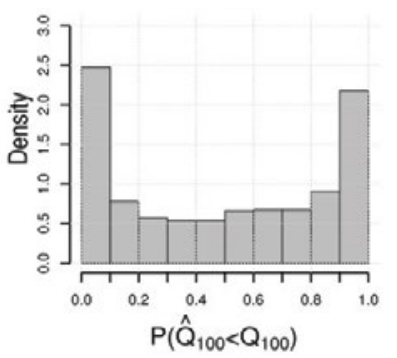

Figure 5 : Distributions des valeurs $\widehat{F} Q_{i}^{(100)}\left(Q_{i}^{(100)}\right)$ obtenues à partir d'échantillons simulés (cf paragraphe II.2.2). a) cas de Saint Martin dans l'Ardèche, et b) cas de Trans en Provence dans le Var (Argens).

d'analyses locales ou régionales (cas $n^{\circ} 2$ et cas $\left.n^{\circ} 4\right)$, permet d'améliorer très sensiblement les performances d'estimation. Les cas des analyses régionales (cas $n^{\circ} 3$ et $n^{\circ} 4$ ) permet en outre de mettre en évidence deux limites importantes inhérentes à ces méthodes :

- tout d'abord, la performance assez limitée des approches basées sur les seules séries hydrométriques (cas 3). Cet effet est dû d'une part à l'imprécision d'estimation des index de crue (moyenne de chaque série), mais aussi surtout à la présence d'hétérogénéités dans la forme des distributions locales des échantillons simulés. Cette hétérogénéité dégrade nettement les performances par comparaison à ce qui avait été identifié dans le cas de régions homogènes [N'guyen et al., 2013]. Finalement, pour les 3 études de cas traités, et compte tenu de l'hétérogénéité supposée des régions c oncernées, les approches régionales classiques offrent des performances assez peu éloignées des approches locales (cas 1). - pour ce qui concerne l'approche valorisant les extrêmes non jaugés, les sources d'imprécision sont essentiellement la présence d'hétérogénéités dans la relation d'index de crue, et dans la forme des distributions locales, avec là encore un effet très significatif de cette deuxième source d'hétérogénéité par rapport aux résultats initiaux présentés dans N'guyen et al. [2013]. Ces deux sources d'erreur sont toutefois assez largement compensées par la présence des données sur les extrêmes, qui représentent l'équivalent d'une très longue période d'observation supplémentaire. Pour les bassins de petite surface (Figure 4.b)), la relation d'index de crue s'avère moins bien déterminée ( $S^{\beta}$ peu sensible à $\beta$ lorsque $S$ est faible), ce qui a pour effet de dégrader fortement 
les performances de cette approche : dans le cas présenté (Saint Laurent dans l'Ardèche), les performances obtenues s'avèrent similaires à celle de l'approche locale.

Les simulations renseignent également sur la valeur à accorder aux intervalles de crédibilité à $90 \%$ issus des approches Bayésiennes MCMC (Figure 5) : s'il apparait que ces intervalles sont correctement estimés dans le cas des approches locales (avec parfois présence d'un léger biais), ceux-ci sont systématiquement sous-estimés dans le cas des approches régionales. Une évaluation similaire, conduite dans les précédents travaux publiés [N'guyen et al., 2013], mais sans prise en compte de l'hétérogénéité des formes des distributions locales, montrait que les intervalles de crédibilité s'avéraient alors bien mieux estimés avec les approches régionales. Finalement, la sous estimation de la largeur des intervalles de crédibilité dans le cas des approches régionales est liée : 1- à la présence d'hétérogénéités régionales dans la forme des distributions, qui ne sont pas prises en compte dans l'estimation des intervalles, et 2- à l'incertitude d'estimation des index de crue (moyenne de chaque série) dans le cas $n^{\circ} 3$, incertitude qui n'est pas non plus prise en compte dans le calcul des intervalles (effet déjà identifié dans N'guyen et al. [2013]). Finalement, c'est donc pour l'approche régionale « classique » basée sur les séries hydrométriques (cas $n^{\circ} 3$ ) que la sous estimation de la largeur des intervalles s'avère la plus marquée.

\section{IV.1.1. Données réelles}

Les résultats d'estimation obtenus à partir des données réelles sont synthétisés dans les Tableau 2 et Tableau 3. Ces résultats laissent apparaitre plusieurs effets qui s'éloignent parfois des indications fournies par les simulations.

Dans le cas de Saint Martin d'Ardèche, la largeur des intervalles de crédibilité se resserre avec les données historiques (approche locale) ou avec l'utilisation des données sur les extrêmes non jaugés (approches régionales), ce qui s'avère cohérent avec les simulations. On peut toutefois remarquer que les quantiles estimés sont significativement différents entre les approches locales et régionales. Une extension de la série historique locale, conduite par Naulet [2005] sur ce site, permet de montrer que la plus forte valeur de la série locale (crue de 1958) a été dépassée 4 fois au cours du XIX'eme siècle en 1827, 1846, 1890 et 1900 . Les débits estimés des crues de 1890 et 1827 ont atteint d'après cette source $7000 \mathrm{~m}^{3} / \mathrm{s}$, valeur très cohérente avec l'estimation du quantile centennal issue des analyses régionales.

Tableau 2 : Résultats d'estimation du quantile centennal à partir des jeux de données réelles, pour les trois sites d'étude.

\begin{tabular}{|c|c|c|c|c|c|c|c|c|c|}
\hline Site & $\operatorname{Cas} n^{\circ}$ & $\mathbf{n}_{\text {cont }}$ & $\mathbf{n}_{\text {hist }}$ & $\mathbf{n}_{\mathrm{reg}}$ & $\mathbf{n}_{\text {ext }}$ & $\widehat{Q}_{100}^{5}$ & $\widehat{Q}_{100}^{M L}$ & $\widehat{Q}_{100}^{95}$ & $\Delta \mathrm{CI}(\%)$ \\
\hline \multirow{3}{*}{ Saint Laurent } & 1 & 30 & & & & 410 & 635 & 1855 & 228 \\
\hline & 3 & 30 & & 138 & & 425 & 520 & 710 & 55 \\
\hline & 4 & 30 & & 138 & 900 & 445 & 500 & 575 & 26 \\
\hline \multirow{4}{*}{ Saint Martin } & 1 & 43 & & & & 3430 & 4040 & 6200 & 69 \\
\hline & 2 & 43 & 50 & & & 3780 & 4300 & 5790 & 47 \\
\hline & 3 & 43 & & 125 & & 5540 & 6810 & 9310 & 55 \\
\hline & 4 & 43 & 50 & 125 & 850 & 5480 & 6560 & 7850 & 36 \\
\hline \multirow{4}{*}{ Trans en Provence } & 1 & 34 & & & & 105 & 145 & 410 & 210 \\
\hline & 2 & 34 & 360 & & & 195 & 265 & 395 & 75 \\
\hline & 3 & 34 & & 215 & & 100 & 110 & 135 & 32 \\
\hline & 4 & 34 & 360 & 154 & 300 & 175 & 210 & 285 & 52 \\
\hline
\end{tabular}

Tableau 3 : Résultats d'estimation du quantile millénal à partir des jeux de données réelles, pour les trois sites d'étude.

\begin{tabular}{|c|c|c|c|c|c|c|c|c|c|}
\hline Site & $\operatorname{Cas} n^{\circ}$ & $\mathbf{n}_{\text {cont }}$ & $\mathbf{n}_{\text {hist }}$ & $\mathbf{n}_{\text {reg }}$ & $\mathbf{n}_{\text {ext }}$ & $\hat{Q}_{1000}^{5}$ & $\hat{Q}_{1000}^{M L}$ & $\hat{Q}_{1000}^{95}$ & $\Delta \mathrm{CI}(\%)$ \\
\hline \multirow{3}{*}{ Saint Laurent } & 1 & 30 & & & & 665 & 1380 & 7680 & 508 \\
\hline & 3 & 30 & & 138 & & 655 & 930 & 1555 & 97 \\
\hline & 4 & 30 & & 138 & 900 & 720 & 870 & 1090 & 43 \\
\hline \multirow{4}{*}{ Saint Martin } & 1 & 43 & & & & 3810 & 5020 & 10060 & 125 \\
\hline & 2 & 43 & 50 & & & 4480 & 5500 & 9060 & 83 \\
\hline & 3 & 43 & & 125 & & 8550 & 12180 & 20320 & 97 \\
\hline & 4 & 43 & 50 & 125 & 850 & 8780 & 11410 & 15400 & 58 \\
\hline \multirow{4}{*}{ Trans en Provence } & 1 & 34 & & & & 140 & 245 & 1375 & 504 \\
\hline & 2 & 34 & 360 & & & 395 & 655 & 1290 & 137 \\
\hline & 3 & 34 & & 215 & & 125 & 150 & 215 & 60 \\
\hline & 4 & 34 & 360 & 154 & 300 & 350 & 485 & 780 & 89 \\
\hline
\end{tabular}


Dans le cas de Saint Laurent, l'estimation est également sensiblement réorientée, cette fois à la baisse, par les approches régionales. Un examen détaillé de la série locale montre que la plus forte valeur de cette série (crue de 1980) apparait comme un horsain : l'incorporation des séries hydrométriques régionales, puis des données sur les extrêmes qui concernent 5 à 10 bassins de taille similaire sur une durée de 50 ans, permettent de confirmer la période de retour élevée de la crue de 1980, et donc le caractère particulier de l'échantillon mesuré à Saint Laurent. Ce cas montre finalement que même si l'intérêt des analyses régionales s'avère en théorie limité (cf. simulations), il peut s'avérer significatif sur un cas particulier, les données régionales mettant ici en évidence le caractère exceptionnel de la crue de 1980 à Saint Laurent.

Dans le cas de Trans-en-Provence, la richesse de la série historique locale réoriente à la hausse les estimations tout en resserrant fortement l'intervalle de crédibilité. L'approche régionale basée sur les séries hydrométriques, de son côté, aboutit à une estimation qui semble anormalement basse : un test statistique permet d'ailleurs de confirmer l'incohérence entre ces estimations et les crues extrêmes de la série historique observée à Trans en Provence. Cet effet est dû à l'absence de crues fortes dans les séries hydrométriques valorisées, ce qui peut être attribué conjointement au hasard de l'échantillonnage mais également à une probable dépendance entre les séries valorisées. La valorisation des données sur les crues extrêmes au sein de l'analyse régionale permet finalement ici de corriger cet effet, et d'aboutir à une estimation beaucoup plus cohérente avec la série observée localement à Trans-en-Provence.

\section{CONCLUSIONS}

Les résultats présentés dans cet article permettent de comparer les performances respectives de méthodes d'analyse fréquentielle des crues basées sur des séries locales et régionales, et incorporant ou non des informations sur les crues extrêmes du site et/ou de la région considérée. Les données relatives aux crues extrêmes valorisées sont liées à des inventaires historiques locaux et/ou à des inventaires régionaux de crues extrêmes sur les sites non jaugés. Ces données complémentaires se présentent sous la forme de séries censurées de longue durée.

L'approche d'évaluation adoptée, basée sur des simulations, permet de comparer les performances respectives de chaque approche (locale et régionale, avec ou sans données sur les extrêmes), en tenant compte à la fois du contexte particulier des jeux de données disponibles dans chaque étude de cas, mais également en s'affranchissant des effets de l'échantillonnage, et en intégrant le respect parfois approximatif des hypothèses d'homogénéité inhérentes aux approches régionales. Ces résultats mettent en évidence d'une part les gains généralisés associés à l'utilisation des jeux de données sur les extrêmes (séries censurées historiques ou régionales), mais également une limitation assez nette des performances des approches régionales en cas de présence d'hétérogénéités. Les approches régionales classiques basées sur les séries hydrométriques voient de plus leurs performances limitées par l'utilisation de la moyenne de chaque série comme estimateur de l'index de crue. Finalement, malgré l'augmentation significative de la longueur des observations valorisées, ces approches peuvent parfois présenter des performances similaires à une simple analyse de la série hydrométrique locale.

Les résultats obtenus sur les jeux de données réels confirment l'intérêt des données sur les crues extrêmes pour relativiser des résultats parfois surprenants et liés à des problèmes d'échantillonnage : confirmation de l'occurrence de crues dépassant celles présentes dans la série étudiée (Trans en Provence et Saint Martin), ou du caractère " exceptionnel » d'une crue apparaissant comme un horsain (Saint Laurent).

Outre la confirmation de l'importance des informations sur les crues extrêmes, ces résultats illustrent l'intérêt de l'approche par simulations pour analyser et relativiser les résultats obtenus à partir des jeux de données réels : largeurs d'intervalles de crédibilité pouvant parfois s'avérer assez fortement sous-estimées, et performances supposée de chaque approche dépendant fortement des effets combinés de la longueur des séries de données disponibles (notamment des séries censurées sur les crues extrêmes), ainsi que du niveau d'hétérogénéité supposé de la région considérée.

\section{REFERENCES}

DALRYMPLE T. (1960) - Flood frequency analyses Water Supply. Geological Survey. Reston, Virginia, USA. 1543-A

Gaume E., Bain V., Bernardara P., Newinger O., Barbuc M., Bateman A., Blaškovicová L., Blöschl G., Borga M., Dumitrescu A., Daliakopoulos I., Garcia J., Irimescu A., Kohnova S., Koutroulis A., Marchi L., Matreata S., Medina V., Preciso E., Sempere-Torres D., Stancalie G., Szolgay J., Tsanis J., Velasco D., Viglione A. (2009) - A Compilation Of Data On European Flash Floods. Journal Of Hydrology. 367 : 70-80

Gaume E., GaÁl L., Viglione A., Szolgay J., Kohnová S., BLÖSCHL G. (2010) - Bayesian MCMC approach to regional flood frequency analyses involving extraordinary flood events at ungauged sites. Journal of Hydrology. 394(1-2) : 101-117

Hosking J., WALlis J. (1993) — Some statistic useful in regional frequency analysis. Water resources research. 29 : 271-281

Hosking J., Wallis J. (1997) - Regional frequency analysis: An approach Based on L-Moments. Cambridge University Press, London, UK

Naulet R., Lang M., Ouarda T.B.M.J., Ceeur D., Bobée B., RECKING A., Moussay D. (2005) — Flood frequency analysis on the Ardèche river using French documentary sources from the last two centuries. Journal of Hydrology. 313 : 58-78

Neppel L., Renard B., Lang M., Ayral P., Coeur D., Gaume E., Jacob N., Payrastre O., Pobanz K., Vinet F. (2010) — Flood frequency analysis using historical data: accounting for random and systematic errors. Hydrological Siences Journal. 55 : 192-208

N'guyen Chi Cong, Payrastre Olivier, Gaume E. (2013) Inventaires de crues extrêmes sur des sites non jaugés et analyse statistique régionale des débits: réflexions méthodologiques et évaluation des performances DOI 10.1051/ lhb/2013011. La Houille Blanche. 2 : 16-23

Payrastre O., Gaume E., And Andrieu H. (2006) - Usefulness of historical flood series reconstruction for the study of extreme floods in small watersheds. Case study of four small tributaries of the Aude river, France. La Houille Blanche. 6 : 79-86

Payrastre O., E. Gaume, And H. Andrieu (2011) Usefulness of historical information for flood frequency analyses: Developments based on a case study, doi10.1029/2010WR009812. Water Resour. Res. 47 : W08511

Payrastre O., E. Gaume, AND H. ANDrieu (2013) — Information historique et étude statistique des crues extrêmes : quelles caractéristiques souhaitables pour les inventaires de crues historiques?, DOI 10.1051/lhb/2013019. La Houille Blanche. 3 : 5-11

Reis D., Stedinger J. (2005) - Bayesian MCMC flood frequency analysis. with historical information. Journal of Hydrology. 313 : $97-116$

Renard B., Garreta V., LANG M. (2006) - An application of Bayesian analysis and Markov chain Monte Carlo methods to the estimation of a regional trend in annual maxima. Water resources research. $\mathbf{4 2 ( 1 2 )}$ : W12422 